\title{
A prospective study of receptor profiles in breast cancer and the ipsilateral axillary lymph node metastases measured simultaneously in treatment naïve cases
}

\author{
A Mannell, ${ }^{1}$ CE Nel, ${ }^{2,3}$ (DS Smilg, ${ }^{4}$ (D) J Haberfield, ${ }^{4}$ S Nietz, ${ }^{1}$ GP Candy ${ }^{1}$ \\ ${ }^{1}$ Department of Surgery, Faculty of Health Sciences University of the Witwatersrand, South Africa \\ ${ }^{2}$ Department of Anatomical Pathology, University of the Witwatersrand, South Africa \\ ${ }^{3}$ National Health Laboratory Services \\ ${ }^{4}$ Department of Radiology, Charlotte Maxeke Johannesburg Academic Hospital, South Africa
}

Corresponding author, email: mannell@mweb.co.za

Background: The heterogeneity of receptor profiles in breast cancer is well known. The differing receptor profiles of primary breast cancer and nodal metastases have been investigated and found to range between 10-50\% depending on the hormone receptor tested. A study comparing the hormone status of primary breast cancers and the synchronous ipsilateral involved sentinel lymph node has not been performed in a South African population.

Method: This is a prospective study where two specialist radiologists performed the simultaneous core needle biopsies of the primary breast cancer and the clinically positive axillary nodes. All receptor status analysis was conducted by one specialist histopathologist.

Results: Of 141 patients who gave written informed consent for this study, 29 were excluded; 112 patients met the inclusion criteria. Anonymised demographics of age, clinical stage, HIV status and metastatic screening were recorded. The simultaneous biopsies and receptor measurements identified 10 patients with discordant receptor status in the positive axillary lymph nodes. In each case, the receptor profile of the axillary lymphatic metastases was more aggressive than that of the primary tumour. The luminal A subtype had a significantly greater risk of discordance than other subtypes $(p=0.02)$.

Conclusion: Core needle biopsy and receptor analysis should be considered on the positive axillary nodes in breast cancer patients. Adjuvant treatment should be targeted to the receptor profile of the lymph node metastases.

Keywords: breast cancer, node metastases, molecular subtypes, discordance

\section{Introduction}

Cancer is a leading cause of death globally, exceeded only by cardiorespiratory diseases, infections, life style disorders and trauma. ${ }^{1}$ Ninety per cent of cancer deaths are due to metastatic spread and global research has intensified in an effort to identify the molecular characteristics which programme cancer cells to metastasise, allow them to evade the immune system, proliferate at distant sites and ultimately overwhelm the patient. ${ }^{2-3}$

In 2000, Perou used global gene expression profiling to identify 5 intrinsic subtypes of invasive breast cancer. Each of these subtypes is unique in incidence, therapeutic response and survival. ${ }^{4}$ As gene expression profiling is not readily available in the public sector, immunohistochemical stains are used as a surrogate to define the molecular subtype of breast cancer. Measuring the hormonal receptors oestrogen receptor (ER), progesterone receptor (PR), human epidermal growth factor receptor 2 (HER2) and the proliferative marker Ki-67 has become critical to the selection of systemic therapy. Breast cancer is a heterogeneous disease and one tumour may contain multiple clones of cells which differ in receptor profiles with the more aggressive subtypes most likely to metastasise. Discordance between the receptor profile of the primary breast cancer and that of the ipsilateral lymph node metastases has been reported. Where the positive axillary lymph nodes exhibit the more aggressive phenotype, targeting the lymphatic metastases as opposed to the primary breast cancer may be indicated. ${ }^{5-8}$

The following is a report of a prospective study of 141 newly diagnosed and treatment naïve patients with breast cancer and ipsilateral axillary node involvement presenting to the Charlotte Maxeke Johannesburg Academic Hospital (CMJAH).

The aim of the study was twofold: first, identification of discordance, in receptor status using the surrogate St Gallen molecular subtypes, between the primary breast cancer and ipsilateral lymph node metastases; second, determination of the impact that phenotype discordance may have on patient management. 


\section{Patients and methods}

This prospective study was conducted on 141 patients presenting to the CMJAH Breast Clinic from July 2017 to December 2018. Informed consent for inclusion in the study was obtained from each patient. Patient data was anonymised and identifying details known only to the primary investigator. This data included age, sex, laterality, routine haematological and serological tests including an Elisa blood test for HIV status. A metastatic screen of bones, liver and lungs was routinely performed.

Four tomosynthesis mammographic views were performed on each patient using a Hologic Selenia Dimensions full view digital machine. "C view" digital 2-D mammograms were generated from tomosynthesis views. The mammograms were viewed on BARCO 5 mega pixel screens. The mammograms and ultrasonography were performed in the mammogram department of the $\mathrm{CMJAH}$ and interpreted by a single team of two diagnostic radiologists, each with over 17 years of experience in breast imaging. Ultrasonography was performed on a Toshiba Xario 100 machine using an 18 $\mathrm{MHz}$ probe.

Core needle biopsies of the primary breast cancer and the ipsilateral lymph node metastases were obtained under sonar guidance using either a Pro Mag 14G biopsy needle or a Super Core 14G biopsy needle provided by Argon Medical Devices. Both the index breast mass and the involved nodes were sampled simultaneously and placed immediately into $10 \%$ neutral buffered formalin. The specimens were processed on the same day undergoing standard overnight processing using an automated Tissue-TEK VIP according to the standard operating procedure of the National Health Laboratory Service laboratory at the CMJAH.

Four micrometer sections were cut off the breast biopsy and the lymph node. The sections were mounted on a glass slide and stained with haematoxylin and eosin using the autostainer, DRS 2000. The pre-treatment of slides for immunohistochemistry is performed using a DAKO automated instrument (PT link), with heat induced epitope retrieval. The following antibodies were used in the laboratory: Anti-Oestrogen receptor rabbit monoclonal primary antibody, Clone SP1: pre-diluted from Roche Diagnostics, USA at high $\mathrm{pH}$; monoclonal mouse antihuman progesterone receptor Clone PgR 636: 1:250 from Dako Denmark at high $\mathrm{pH}$; polyclonal rabbit anti-human c-erbB-2 oncoprotein: Code A0485, 1:500 from Dako Denmark at low pH; monoclonal anti-human Ki-67 antigen Clone MIB-1 Code M7240, 1:100 from Dako Denmark at high $\mathrm{pH}$.

All cases were reviewed by the same specialist histopathologist and reported on simultaneously.

The presence of invasive cancer was confirmed in the samples from the breast and lymph node, classified according to the morphological type and the Nottingham Grading System used to grade the tumours. ${ }^{8}$ The Allred score was used in ER and PR measurement. ${ }^{9}$ Fluorescent In Situ Hybridisation (FISH) was performed when immunohistochemical analysis of the HER2 receptor gave an equivocal HER2 result. For HER2 measurement, tumours scored as $1^{+}$on IHC were considered negative and those scoring $3^{+}$as positive. The fraction of proliferating cells positive for $\mathrm{Ki}-67$ was calculated from a count of at least 500 cancer cells and expressed as a percentage. ${ }^{10}$
Based on the criteria recommended by the St Gallen International Expert Consensus Report 2013, receptor profiles were classified as follows: ${ }^{11}$

Luminal A: ER positive or PR positive and HER2 negative with a Ki-67 of $\leq 20 \%$

Luminal B: ER positive or PR positive and HER2 negative with a Ki-67 of $>20 \%$.

Luminal HER2 positive: ER positive, HER2 over-expressed or amplified, with any PR and any Ki-67.

Non-luminal HER2 enriched: ER negative and PR negative but HER2 positive with any Ki-67.

Triple negative: ER negative, PR negative, HER2 negative.

\section{Statistical analysis}

The statistical analysis was performed with STATISTICA Software Version 13.5. Chi-squared test with Fisher's exact test for small numbers $<5$ was used to examine the associations of HIV status, clinical stage, molecular subtypes and $\mathrm{Ki}-67 \%$ with rate of discordance between the receptor profiles of primary breast cancer and the metastases. A $p$-value of $<0.05$ between groups was regarded as significant.

\section{Results}

From July 2017 to December 2018, 141 patients with newly diagnosed, treatment naïve breast cancer were seen at the CMJAH Breast Clinic and considered eligible for this study of the receptor profile of the primary breast cancers and synchronous ipsilateral lymph node metastases. Of a total of 141 patients, 29 cases were excluded when the biopsies yielded insufficient necrotic or benign tissue, and when the primary was either a non-epithelial malignancy, a recurrent cancer or it was discovered that the patient had received neoadjuvant therapy prior to presentation. A total of 112 patients were entered on this study. Of these, 110 patients had a unilateral primary breast cancer and 2 patients presented with simultaneous bilateral breast cancer.

The patient demographics, laterality of the primary breast cancer, clinical staging and HIV status are detailed in Table 1. There were 110 women and 2 men with a mean age of 54 (range 31-81). The tumours were equally distributed between right and left breasts: two patients had bilateral

\begin{tabular}{lc} 
Table 1: Patient demographics & \\
\hline Age & \\
54 years $(\mathrm{SD} \pm 13)$ & \\
\hline Gender & 2 \\
Male & 110 \\
Female & \\
\hline Laterality & 57 \\
Right & 53 \\
Left & 2 \\
Bilateral & \\
\hline HIV status & 88 \\
Negative & 15 \\
Positive & 9 \\
Unknown & \\
\hline Clinical stage & 0 \\
I & 7 \\
II & 78 \\
III & 27 \\
IV & 112
\end{tabular}


Table 2: Histological subtypes and grade

\begin{tabular}{lcc} 
Histological subtype & Breast & Nodes \\
\hline Ductal carcinoma & 103 & 103 \\
Lobular carcinoma & 4 & 4 \\
Other* & 5 & 5 \\
& & \\
Grade & 6 & 5 \\
1 & 58 & 60 \\
2 & 48 & 47 \\
3 & & \\
*Includes mucinous carcinoma, metaplastic carcinoma and carcinoma with \\
apocrine differentiation.
\end{tabular}

primary breast cancer. Fifteen patients (13\%) were HIV positive, $88(79 \%)$ negative and 9 unknown. The majority (79\%) had stage III disease, 7 were clinically stage II and 26 had distant disease. The histological subtypes and grade of the primary tumour and the lymph node metastases are listed in Table 2.

Table 3 lists the molecular subtypes and the rate of discordance in each subtype between the breast and lymph nodes. Forty-one primary breast cancers exhibited a luminal $B$ profile with an equal number of cases either luminal A (22) or triple negative (22). The HER2 receptor was positive in 27 of which 11 were luminal B HER2 enriched and 16 nonluminal HER2 enriched. The highest rate of discordance (27\%) was found in the luminal A subtype.

Table 4 documents the changing receptor profiles in each of the discordant cases. All nine patients exhibited a more aggressive molecular subtype in the lymphatic metastases, with 3 patients expressing HER2 in the lymph nodes and another 3 patients with triple negative lymphatic metastases.

When the rate of discordance in each of the molecular subtypes was analysed, discordance was significantly greater in luminal A compared to luminal B $(p=0.0019)$ and all other molecular subtypes combined $(p=0.0005)$ There was, however, no association between discordance and HIV status ( $p=0.28$ ) nor with clinical stage, where no trend could be observed due to the small number of stage II and IV cases. This analysis failed to find a statistically significant association between $\mathrm{Ki}-67$ and molecular subtype discordance $(p=0.06)$. Using the Fischer's exact test, a significant $p$-value of 0.02 was found between the discordance rate in the luminal A subgroup compared to all the other subtypes (Table 5).

\section{Discussion}

In this study, there was discordance between the receptor profile of the breast cancer and the lymph node metastases in $9 \%$ of patients. In each of these, the lymph node metastases exhibited a more aggressive molecular subtype than the primary, a phenomenon noted by earlier workers..$^{5-7}$

Various explanations have been offered for this discordance: these include technical difficulties which affect the accuracy of the assays. ${ }^{12}$ Commonly, these problems relate to the type and duration of tissue fixative, the choice of antibodies and the stains used in the immunohistochemical techniques as well as lack of FISH for equivocal HER2 receptor measurement. Not only is discordance evident when laboratory results are compared to those of a central accreditation service, but results may differ between histopathologists. This observer difference is most likely to occur when scoring systems include subjective assessment of the intensity of immunohistochemical stains such as the Allred score for ER measurement 9. In addition, when retrospective receptor studies are undertaken, time delay can result in discordance between the two sets of results. ${ }^{13}$

To eliminate technical variations in receptor profiling the current study was prospective, and performed over a limited time frame of 18 months (July 2017 to December 2018). The biopsies were taken simultaneously by a single team of 2 experienced specialist radiologists and placed immediately into a standard solution of $10 \%$ buffered formalin. The receptor assays were performed at a single tertiary institution

Table 3: Molecular subtypes with percentage discordance

\begin{tabular}{lcccc}
\hline & Breast & Lymph node & Discordance & $p$-values \\
\hline Luminal A & 22 & 14 & $27 \%$ & 0.0106 \\
Luminal B & 41 & 44 & $7 \%$ & 0.50 \\
Luminal HER2 enriched & 11 & 12 & $9 \%$ & 0.50 \\
Triple negative & 22 & 25 & $14 \%$ & 0.50 \\
Non-luminal HER2 enriched & 16 & 16 & $0 \%$ & -
\end{tabular}

\begin{tabular}{|c|c|c|c|c|}
\hline Case number & Stage & Breast & & Lymph nodes \\
\hline 14 & III & Luminal A & $\rightarrow$ & Luminal HER2 enriched \\
\hline 25 & III & Luminal A & $\rightarrow$ & Luminal B \\
\hline 56 & III & Luminal A & $\rightarrow$ & Luminal HER2 enriched \\
\hline 63 & III & Luminal B & $\rightarrow$ & Triple negative \\
\hline 72 & III & Luminal A & $\rightarrow$ & Luminal B \\
\hline 91 & IV & Luminal A & $\rightarrow$ & Luminal B \\
\hline 92 & III & Non-luminal HER2 enriched & $\rightarrow$ & Triple negative \\
\hline 104 & III & Triple negative & $\rightarrow$ & Non-luminal HER2 enriched \\
\hline 107 & IV & Luminal A & $\rightarrow$ & Luminal B \\
\hline Rate discordance & & $10 / 112$ & $\cong$ & $9 \%$ \\
\hline
\end{tabular}

Table 5: Comparison of discordant vs concordant cases in different molecular subgroups

\begin{tabular}{lccccc}
\hline \multicolumn{7}{l}{ Table 5: Comparison of discordant vs concordant cases in different molecular subgroups } \\
\hline & Luminal A & Luminal B & Luminal HER2 & Triple negative & Non-luminal HER2 \\
\hline Concordant cases & 16 & 40 & 10 & 21 & 16 \\
Discordant cases & 6 & 1 & 0 & 1 & 1
\end{tabular}


where the laboratory techniques are accredited annually by the South African National Accreditation Service. To eliminate inter observer difference, a single specialist histopathologist measured the receptors in both biopsies simultaneously: when the assay of the HER2 receptor gave equivocal results, FISH was used in all cases to identify true positivity.

It is, therefore, reasonable to assume that the receptor assays in the current study represent the St Gallen molecular subtypes in the primary breast cancer and the ipsilateral lymph node metastases. None of these newly diagnosed primary breast cancer patients entered on this study had received local or systemic treatment: thus the discordant molecular subtypes in the ipsilateral lymph node metastases cannot be attributed to clonal selection of breast cancer cells resistant to previous therapies. ${ }^{13}$ The discordance observed in this study is most likely due to tumour heterogeneity where genetically different cell populations have evolved within the primary tumour. ${ }^{14}$ This complex clonal genomic evolution can result in a change of malignant phenotype and acquisition of metastatic potential. ${ }^{13}$

In this study, 105 patients were diagnosed with advanced breast cancer. Seven patients presented with early stage II disease. This small number of early breast cancer cases did not allow identification of a significant association between tumour volume and receptor discordance. Of the patients with receptor discordance, there were 2 patients (cases 19 and 107 see Table 5) with stage IV disease.

In Table 4, the discordance between the breast cancer and the metastases exposed additional receptors for systemic therapy targeted to the nodal metastases: in 3 patients gain of HER 2 receptor mandates trastuzumab therapy which will extend survival and improve disease control overall. ${ }^{15}$

The pace of global research to identify malignant metastatic pathways in the breast cancer cell has quickened. New agents such as CDK 4/6 inhibitors which block the cell cycle have been developed. ${ }^{16}$ In the current study, 18 luminal tumours evolved in the lymph nodes to more aggressive subtypes with increased rates of proliferation (Ki-67 > 20\%). ${ }^{10}$ These lymphatic metastases are likely to have greater potential for distant spread. It is this proliferation of breast cancer at distant sites which overwhelms the metabolism of the individual with fatal results in $30 \%$ of early breast cancer patients. ${ }^{2}$ Addition of new agents to standard chemotherapy regimens should be considered for these cases.

There was concordance between the molecular subtype of the primary and the node metastases in $91 \%$ patients. In a similar prospective study comparing the molecular subtypes in breast cancer with those of nodal metastases, concordance was $89 \% .{ }^{17}$ In $11 \%$ of patients the molecular subtype in the lymph node displayed a more aggressive subtype than the primary breast cancer. The predominant molecular subtype in this Swedish Study of Breast Cancer patients was luminal A which, as in the current study, had the greatest rate of discordance compared to the other subtypes. ${ }^{17}$ In an older retrospective study of ER and HER2 receptors using archived tissue from primary breast cancer and synchronous node metastases, a similar rate of concordance $(83 \%)$ was identified. ${ }^{18}$ However with quantitative fluorescence, other workers have shown that a significant number of patients exhibit discordance in the amount of receptor protein between primary breast tumour and paired nodal metastases. ${ }^{19}$
This discordance in the quantity of receptor expression may offer an explanation for limited responses to targeted therapies. Whether there is a link between discordance in quantitative receptor expression and the potential for distant spread remains uncertain.

\section{Conclusion}

In this study of the molecular subtypes in the primary breast cancers and the positive ipsilateral axillary lymph nodes, there was discordance in the receptor profiles of 9 patients. In each case, the molecular subtype in the lymphatic metastasis was more aggressive than that of the primary tumour: 3 patients gained HER2, another 2 had triple negative lymph nodes with the final 4 displaying an increase in the rate of proliferation in the nodes. Analysis of the receptors of lymph node metastases is strongly recommended: systemic therapy should target lymphatic metastases to reduce the risk of distant lymphatic spread.

\section{Conflict of interest}

The authors declare no conflict of interest.

\section{Funding source}

This study was funded by the Department of Education, University of the Witwatersrand.

\section{Ethical approval}

Human Research Ethics Committee (Medical), University of the Witwatersrand (Certificate No M170425).

\section{ORCID}

A Mannell (D) https://orcid.org/0000-0003-4438-4045

CE Nel (D) https://orcid.org/0000-0002-3472-9241

JS Smilg (iD https://orcid.org/0000-0002-2406-1436

S Nietz (iD https://orcid.org/0000-0002-6313-2794

GP Candy (iD https://orcid.org/0000-0002-5449-1277

\section{REFERENCES}

1. Torre LA, Siegal RL, Ward EM, Jemal A. Global cancer incidence and mortality rates and trends - an update. Cancer Epidemiol Biomarkers Prev. 2016 Jan;25(1):16-27. https:// doi.org/10.1158/1055-9965.EPI-15-0578. Epub 2015 Dec 14.

2. Martin TA, Ye L, Sanders AJ, et al. Cancer invasion and metastasis: molecular and cellular perspective. In: Madame Curie Bioscience Database Internet. Austin (TX): Landes Bioscience; 2000-2013. Available from: https://www.ncbi. nlm.nih.gov/books/NBK164700/.

3. Sopik V, Narod SA. The relationship between tumour size, nodal status and distant metastases: on the origins of breast cancer. Breast Cancer Res Treat. 2018 Aug;170(3):647-56. https://doi.org/10.1007/s10549-018-4796-9.

4. Perou CM, Sorlie T, Eisen MB, et al. Molecular portraits of human breast tumours. Nature. 2000;406:747-52.

5. Rossi S, Basso M, Strippoli A. Hormone receptor status in primary breast cancer compared with synchronous axillary metastases or recurrent metastatic disease. Clin Breast Cancer. 2015 Oct;15(5):307-12. https://doi.org/10.1016/JSL bc.2015.03.010.

6. Aitken SJ, Thomas SP, Langdon DJ, et al. Quantitative analysis of changes in ER, PR and HER2 expression in primary breast cancer and paired nodal metastases. Ann Oncol. 2010;21:1254-61. 
7. El Nemr Esmail RS, El Farouk Abdel-Salam LO, Abd El Ellah MM. Could the breast prognostic biomarker status change during disease progression? An immunohistochemical comparison between primary tumours and synchronous nodal metastasis. Asian Pac J Cancer Prev. 2015;16(10):4317-21.

8. Rakha EA, Reis-Filho JS, Baehmer F, et al. Breast cancer prognostic classification in the molecular era. The role of histological grade. Breast Cancer Res. 2010;12(4):207. https://doi.org/10.1186/bcr2607. Epub 2010 Jul 30.

9. Allred DC, Harvey JM, Berardo M, Clark GM. Prognostic and Predictive factors in breast cancer by immunohistochemical analysis. Mod Pathol. 1998 Feb;11(2)155-68.

10. Bustreo S, Osella-Abate S, Cassoni P, et al. Optimal Ki67 cutoff for luminal breast cancer prognostic evaluation: a large case series study with a long-term follow-up. Breast Cancer Res Treat. 2016;157(2):363-71. https://doi.org/10.1007/ s10549-016-3817-9.

11. Ignatiadis M, Buyse M, Sotiriou C. St Gallen International Expert Consensus on the primary therapy of early breast cancer: an invaluable tool for physicians and scientists, Ann Oncol. 2015Aug;26(8):1519-20. https://doi.org/10.1093/ annonc/mdv259.

12. Dowsett M, Nielsen TO, A'Hern R, et al. Assessment of Ki67 in breast cancer: recommendations from the International Ki67 in Breast Cancer working group. J Natl Cancer Inst. 2011;103(22):1656-64. https://doi.org/10.1093/jnci/djr393.

13. Pusztai L, Viale G, Kelly CM, Hudis CA. Estrogen and HER-2 receptor discordance between primary breast cancer and metastasis. Oncologist. 2010;15(11):1164-8. https://doi. org/10.1634/theoncologist.2010-0059.
14. Torres L, Ribeiro FR, Pandis N, Andersen JA, Heim S, Teixeira MR. Intratumor genomic heterogeneity in breast cancer with clonal divergence between primary carcinomas and lymph node metastases. Breast Cancer Res Treat. 2007 Apr;102(2):143-55. https://doi.org/10.1007/s10549-0069317-6.

15. Von Minckwitz G, Schneeweiss A, Loibl S, et al. Neoadjuvant carboplatin in patients with triple-negative and Her2 positive early breast cancer (GeparSixto; GBG 66): a randomized phase 2 trial. Lancet Oncol. 2014 Jan:15(7):747-56. https:// doi.org/10.1016/S1470-2045 (14)701-60-3.

16. Matutino A, Amaro C, Verma S. CDK4/6 inhibitors in breast cancer: beyond hormone receptor-positive HER2-negative disease. Ther Adv Med Oncol. 2018;10:1758835918818346. Epub 2018 Dec 17. https://doi. org/10.1177/1758835918818346.

17. Falck AK, Ferno M, Bendahl PO, et al. St Gallen molecular subtypes in primary breast cancer and matched lymph node metastases - aspects on distribution and prognosis for patients with Luminal A tumours: result from a prospective randomised trial. BMC Cancer. 2013 Nov;13(1):558-68.

18. Jensen JD, Knoop A, Ewertz M, Laenkholm AV. ER, Her 2 and TOP2A expression in primary tumour, synchronous axillary nodes, and asynchronous metastases in breast cancer. Breast Cancer Res Treat. 2012 Apr;132(2):511-21. https://doi. org/10.1007/s10549-011-1610-3.

19. Aitken SJ, Thomas JS, Langdon SP, Harrison DJ, Faratian D. Quantitative analysis of changes in EP, PR and Her2 expression in primary breast cancer and faired nodal metastases. Ann Oncol. 2010 Jun;21(6):1254-61. https://doi. org/10.1093/annonc/mdp427. 